\title{
An Autonomous Laser Kirigami Method With Low- Cost Real-Time Vision-Based Surface Deformation Feedback System
}

\section{Zhujiang Wang}

Ontario Tech University

Zimo Wang ( $\sim$ zimowang@binghamton.edu )

SUNY Binghamton: Binghamton University https://orcid.org/0000-0001-9667-0313

\section{Woo-Hyun Ko}

Texas A\&M University

Ashif Sikandar lquebal

Arizona State University

\section{Vu Nguyen}

Lawrence Berkeley National Laboratory Berkeley

Nazanin Afsar Kazerooni

Texas A\&M University

\section{Qiyang Ma}

State University of New York at Binghamton

\section{Arun Srinivasa}

Texas A\&M University

\section{Panganamala Ramana Kumar}

Texas A\&M University

\section{Satish Bukkapatnam}

Texas A\&M University

\section{Research Article}

Keywords: Cyber-physical manufacturing process, Laser kirigami process, Smart manufacturing

Posted Date: April 27th, 2021

DOl: https://doi.org/10.21203/rs.3.rs-416089/v1

License: (c) (1) This work is licensed under a Creative Commons Attribution 4.0 International License.

Read Full License 
Version of Record: A version of this preprint was published at The International Journal of Advanced Manufacturing Technology on September 23rd, 2021. See the published version at https://doi.org/10.1007/s00170-021-07661-8. 


\section{Abstract}

We introduce an autonomous laser kirigami technique, a novel custom manufacturing machine system which functions somewhat similar to a photocopier. This technique is capable of creating functional freeform shell structures using cutting and folding (kirigami) operations on sheet precursors. Conventional laser kirigami techniques are operated manually and rely heavily on precise calibrations. However, it is unrealistic to design and plan out the process (open loop) to realize arbitrary geometric features from a wide variety of materials. In our work, we develop and demonstrate a completely autonomous system, which is composed of a laser system, a 4-axis robotic arm, a real-time vision-based surface deformation monitoring system, and an associated control system. The laser system is based on the Lasersaur, which is a 120 -Watt $\mathrm{CO}_{2}$ open source laser cutter. The robotic arm is employed to precisely adjust the distance between a workpiece and the laser lens so that a focused and defocused laser beam can be used to cut and fold the workpiece respectively. The four-axis robotic arm provides flexibility for expanding the limits of possible shapes, compared to conventional laser machine setups where the workpiece is fixed on rigid holders. The real-time vision-based surface deformation monitoring system is composed of four low-cost cameras, an integrated Al-assisted algorithm, and the sensors (detachable planar markers) mounted on the polymer-based sheet precursors, and allows real-time monitoring of the sheet forming process and geometric evolution with a geometric feature estimation error less than $5 \%$ and delay time around $100 \mathrm{~ms}$. The developed control system manages the laser power, the laser scanning speed, the motion of the robotic arm based on the designed plan as well as the close-loop feedback provided by the vision-based surface deformation monitoring system. This cyber-physical kirigami platform can operate a sequence of cutting and folding processes in order to create kirigami objects. Hence, complicated kirigami design products with various different polygonal structures can be realized by undergoing sequential designed laser cuts, and bends (at any folding angles within designed geometric tolerance) using this autonomous kirigami platform.

\section{Full Text}

This preprint is available for download as a PDF.

\section{Figures}




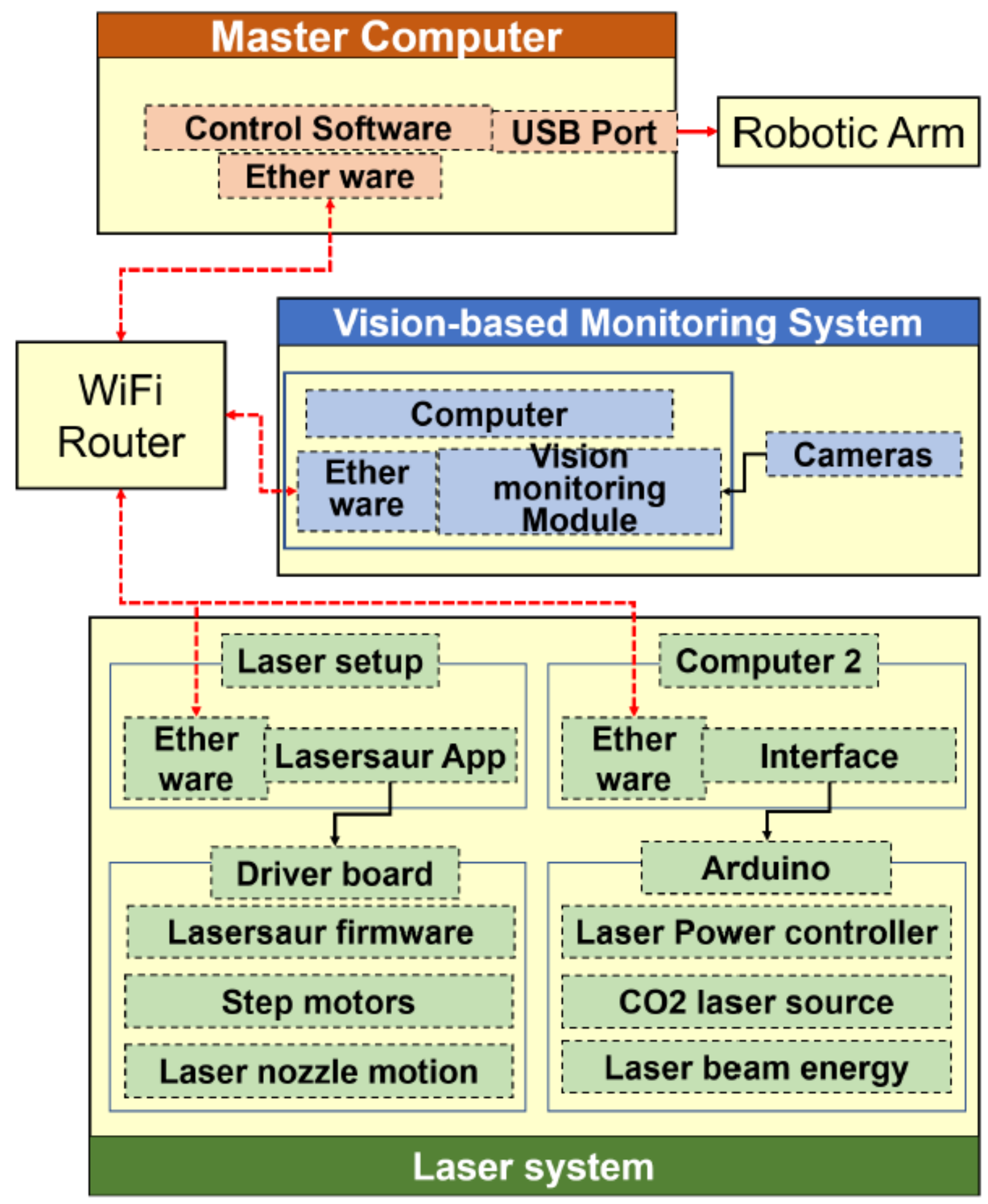

Figure 1

The autonomous kirigami system consists of three subsystems: a control system (master computer), actuators (robotic arm and laser system), and a real-time vision-based monitoring system. A WiFi router is employed to establish the communication among the master computer, the laser system, and the visionbased monitoring system through Etherware networking. The master computer can control the laser 
beam power and the motion of the laser head as well as the robotic arm to conduct the kirigami processes (cutting and folding), with the feedback provided by the vision monitoring system.

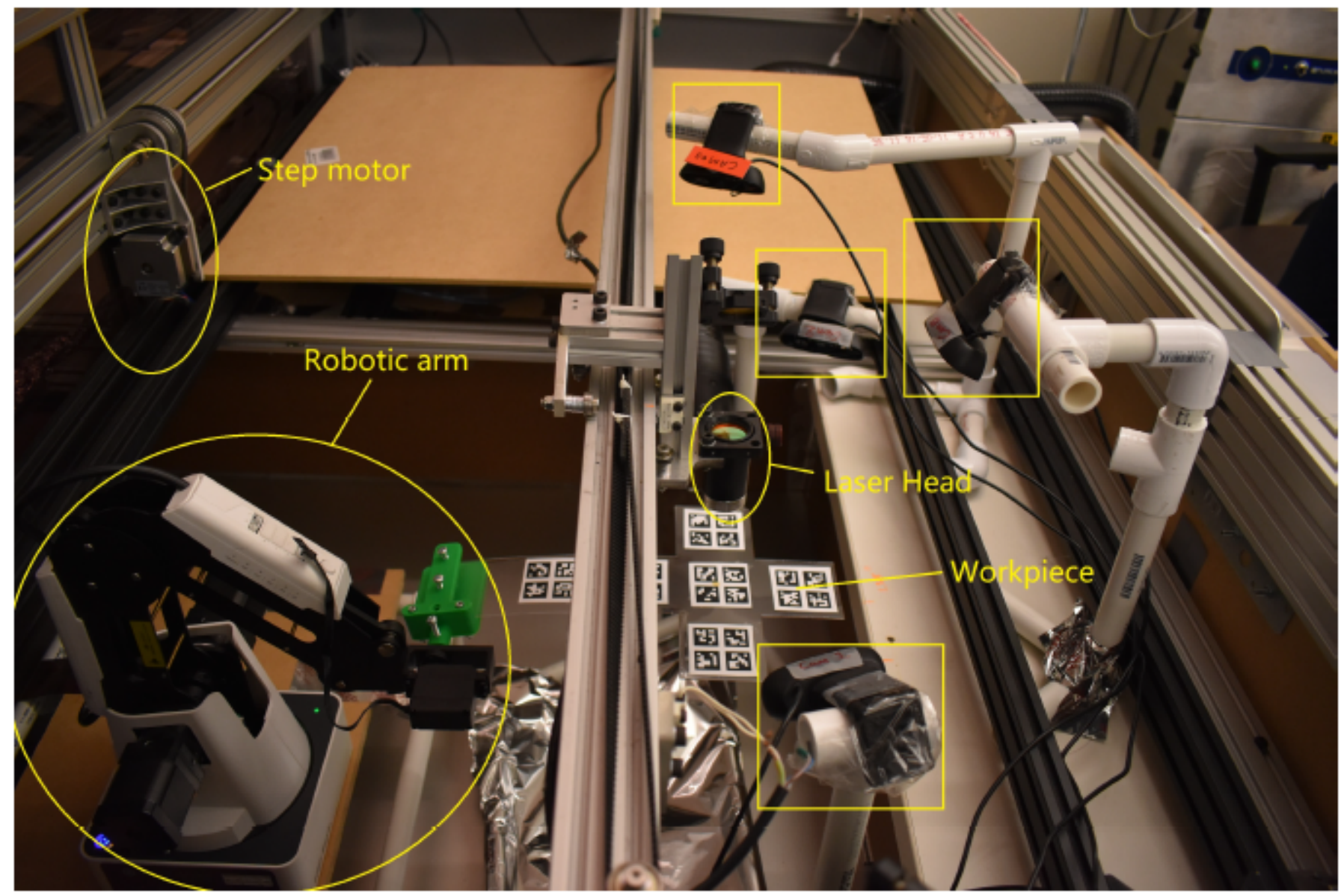

Figure 2

The robotic arm holds a workpiece and can change its position and orientation. The laser head can move horizontally. The four low-cost cameras (highlighted by rectangles) of the vision based surface deformation measurement system are distributed around the workpiece.

(a)

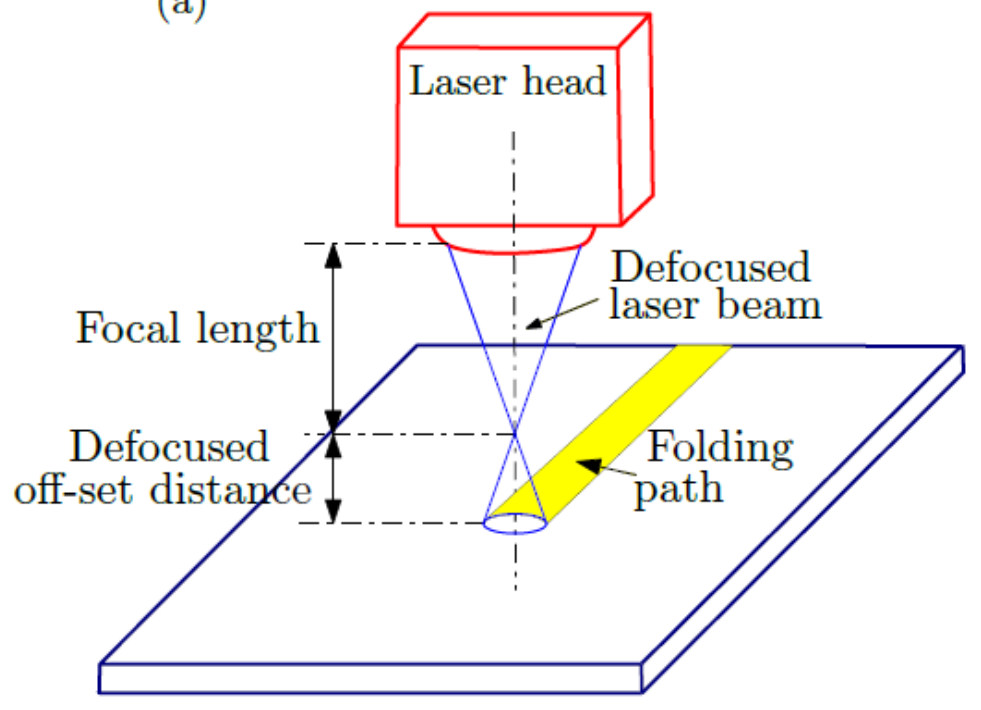

(b)

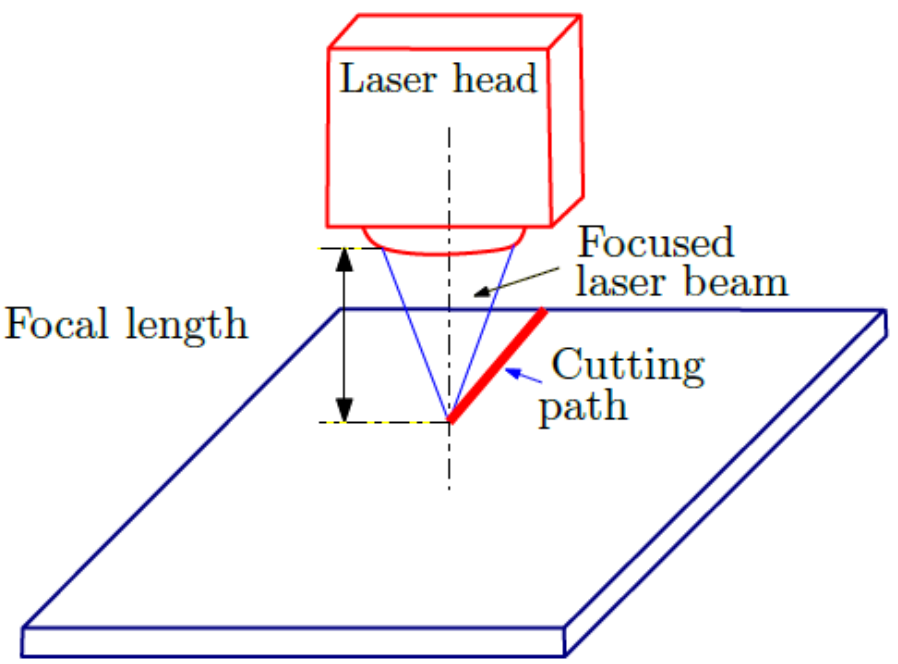




\section{Figure 3}

(a) The distance between the laser lens and a workpiece is greater than the laser lens' focal length, the laser beam is defocused and heats the workpiece along a designed folding path; when the temperature of the workpiece along the folding path surpasses the glass transition temperature, the sheet precursor becomes so soft that it folds under gravity. (b) When the distance is equal to the laser lens' focal length, the laser beam is well-focused on the region of incidence on the workpiece and thus cuts the workpiece. In our system, we use a 4-axis robotic arm holding the workpiece in order to adjust the distance between the laser lens and the workpiece.

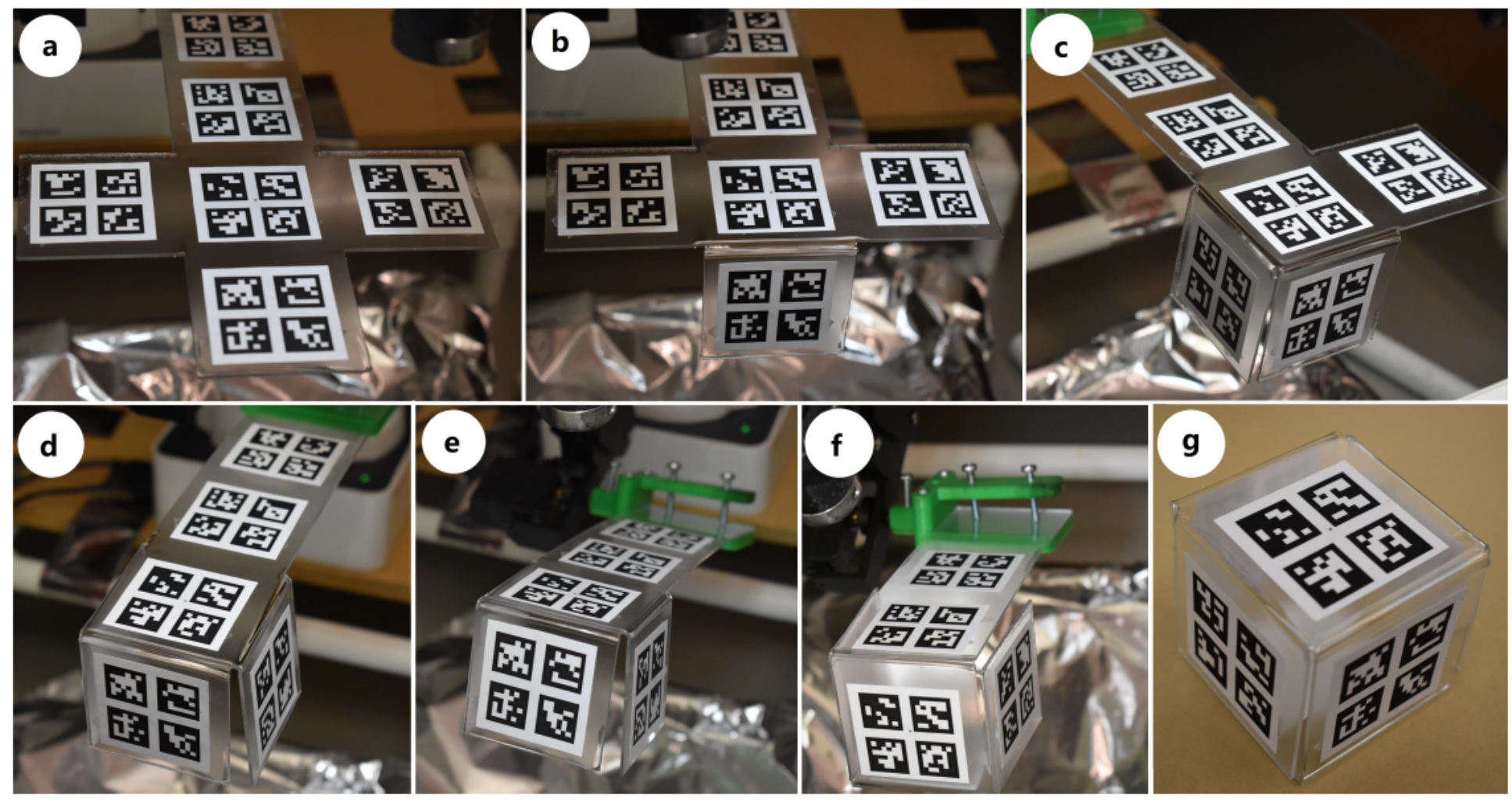

Figure 4

The sequential folding processes for a kirigami box could be achieved through the followings operations: (a) clamp the previously cut 2D sheet precursor with markers on the robotic arm; use defocused laser beam to heat the workpiece along the folding paths 1,2, and 3 (see Fig. 11) to bend the sheet into the desired shape in (b), (c), and (d) respectively; slightly rotate the workpiece by $45^{\circ}$ towards the laser nozzle, and heat the folding path 4 to create the shape in (f); heat the folding path 5 of the shape in (f) and then cut the workpiece along the cutting path 6 to form a closed box (g). 


\section{Folding Sequence based on Kirigami Design}

Kirigami Design with planar marker
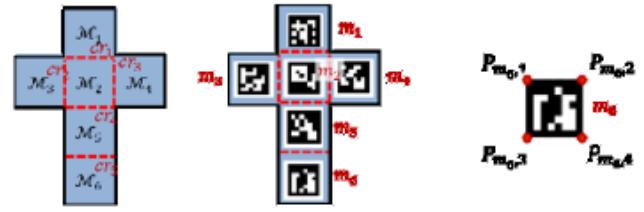

Generated Process Table

Folding sequence design

\begin{tabular}{c|c|c|c}
\multicolumn{4}{c}{ Generated Process Table } \\
\cline { 2 - 4 } Process No. & Crease No. & Facet No. & Dihedral Angle \\
\hline 1 & $l(1)$ & $M_{1}, M_{3}$ & $\theta_{12}=90^{\circ}$ \\
2 & $l(2)$ & $M_{1}, M_{4}$ & $\theta_{14}=90^{\circ}$ \\
3 & $l(3)$ & $M_{1}, M_{2}$ & $\theta_{12}=90^{\circ}$ \\
$\ldots$ & $\ldots$ & $\ldots$ & $\ldots$ \\
\hline
\end{tabular}
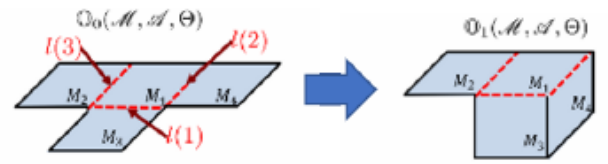

\section{Vision Sensors for Process Monitoring}

Selected vision sensors for sheet bending monitoring
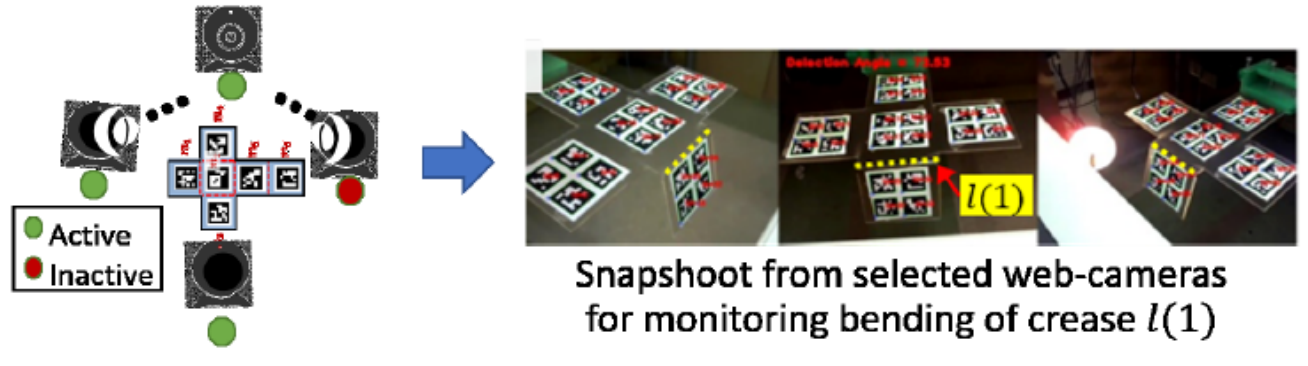

Pose Estimation and projection mapping
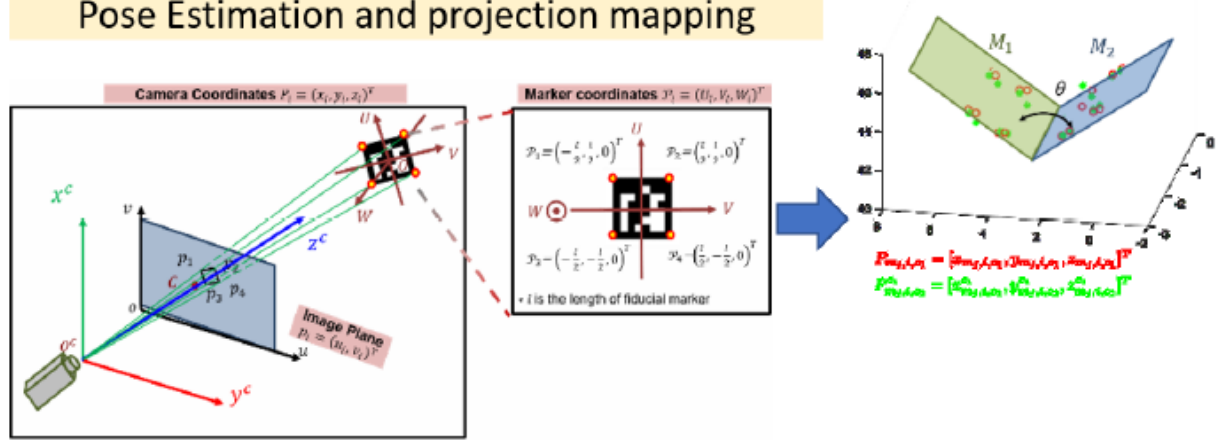

\section{Figure 5}

A schematic diagram showing the vision based real-time monitoring for kirigami sheet bending process. 


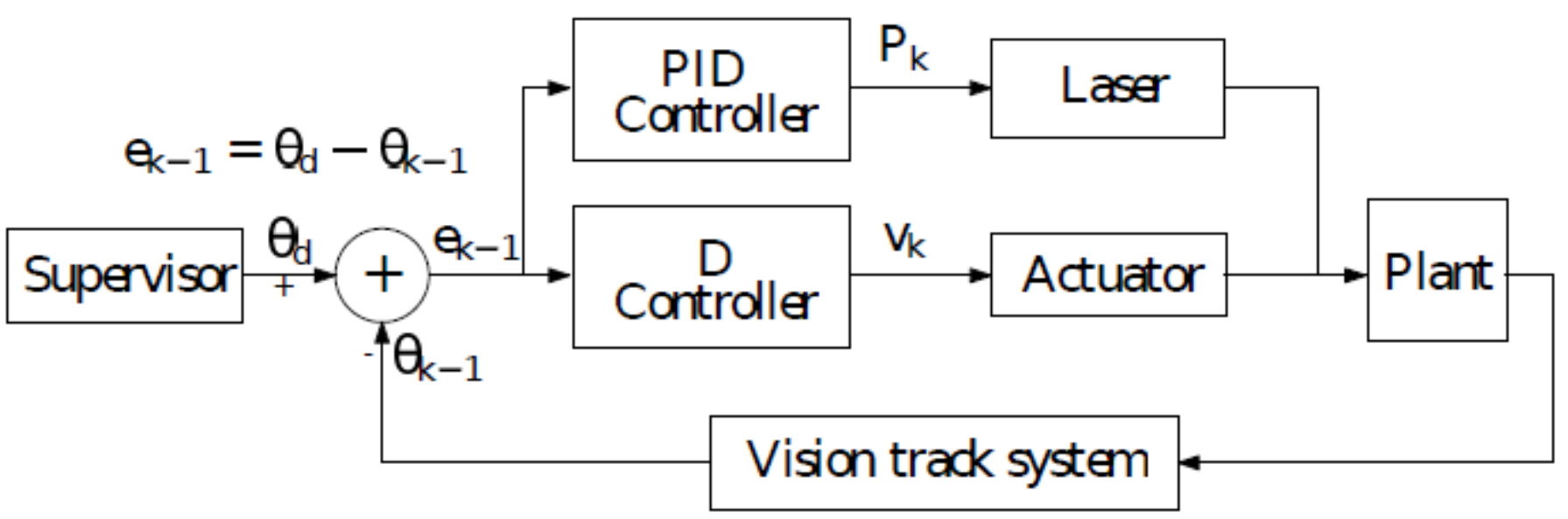

Figure 6

The core logic controlling the defocused laser beam to bend workpieces, where $\theta k-1$ is the observed bending angle, $\theta \mathrm{d}$ the desired bending angle, Pk the laser power, and $\mathrm{vk}$ the laser scanning speed.
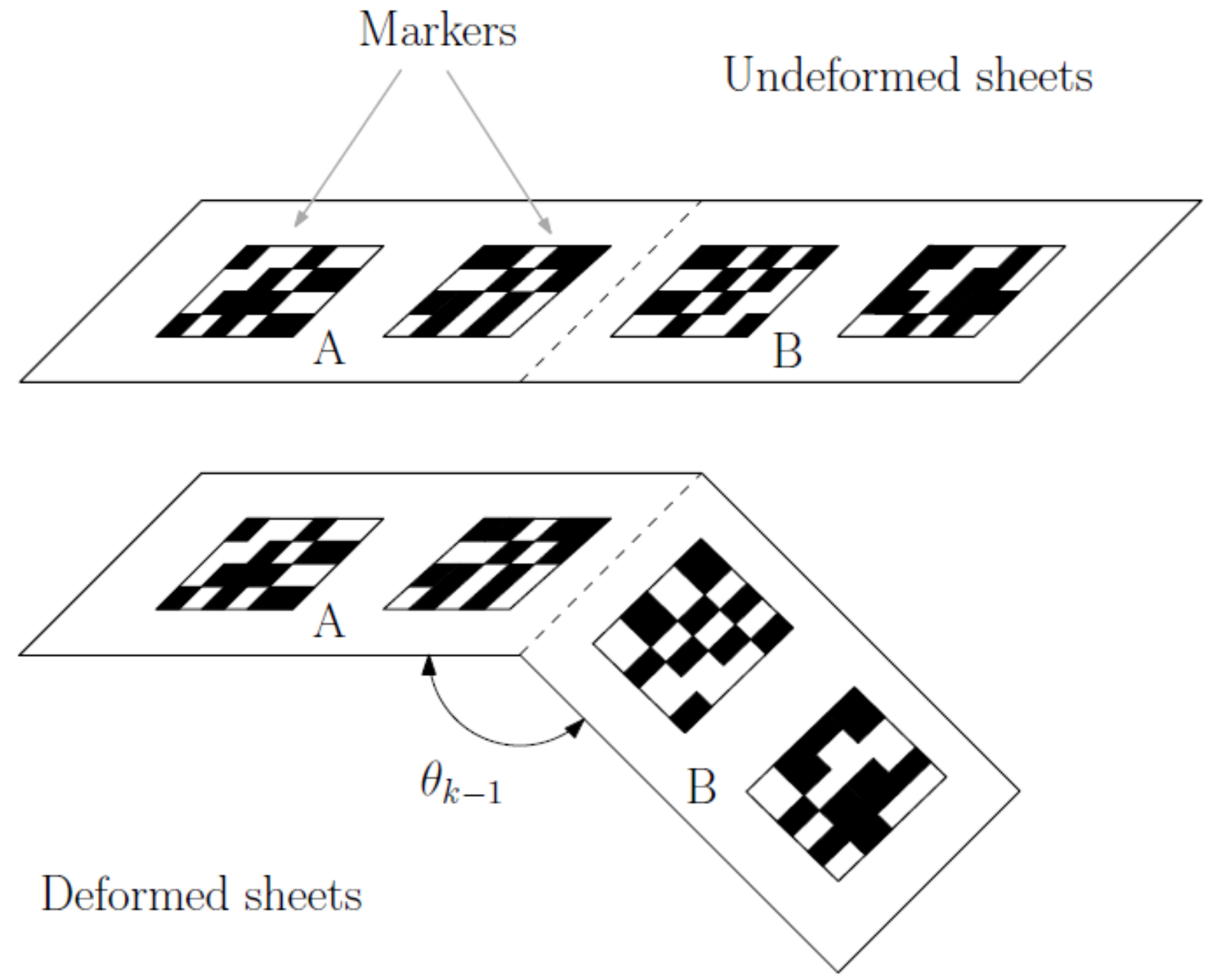
Figure 7

The sheet forming process: the top figure shows the planar sheet with markers pasted on its surface at the initial step. The defocused laser beam scans and heats the workpiece following the designed folding path represented by the dashed line; then the part around the dashed line becomes so soft that the gravity of facet $B$ can bend the sheet.

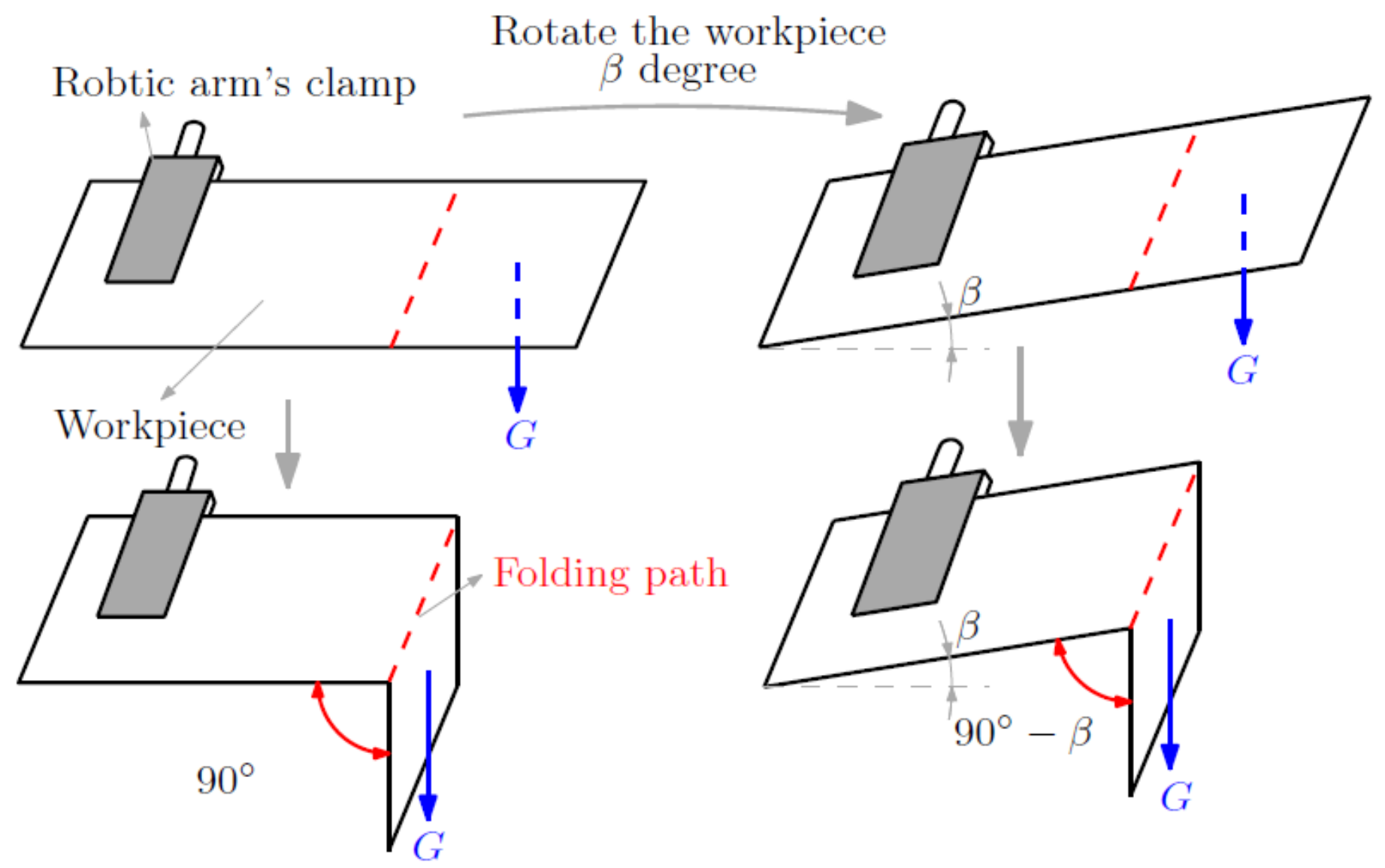

Figure 8

The workpiece is horizontally clamped at an initial step; the dashed line represents the folding path; the gravity $\mathrm{G}$ can fold the workpiece when the laser heats the folding path. If the workpiece is kept in a horizontal plane, the maximum bending angle is $90^{\circ}$ (left); if the robotic arm rotates the workpiece by $\beta$ degrees, the maximum bending angle can be extended to $\beta+90^{\circ}$ (right). 


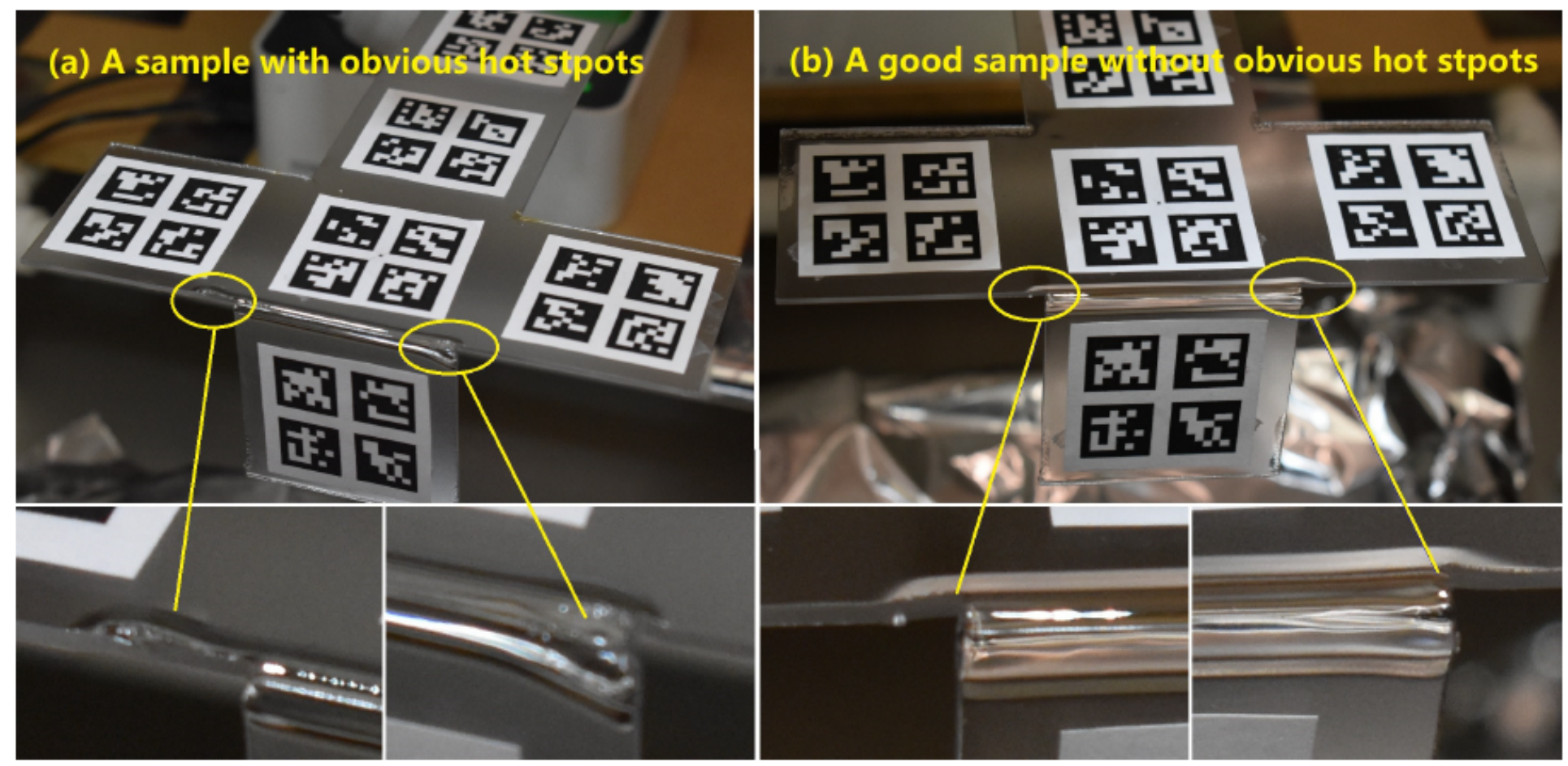

Figure 9

(a) The hot spots cause the melting areas around two ends of the crease when the laser scanning path precisely follows the folding path. (b) A good sample without noticeable melting points results when the laser scanning path follows the scheme shown in Fig. 10. 


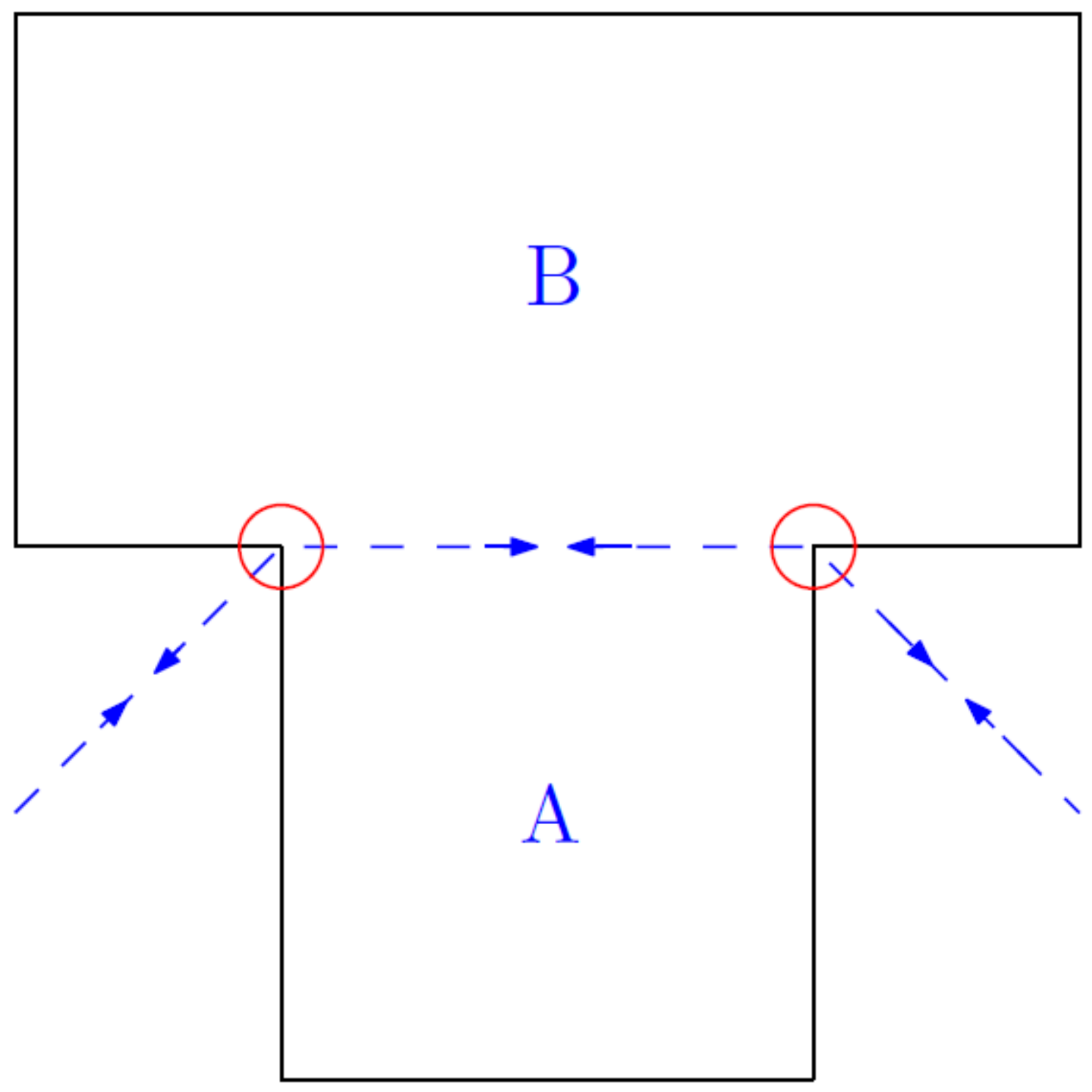

Figure 10

The dashed line shows a proper laser scanning path. This path can effectively avoid the melting points highlighted by two circles. 


\section{Top View (initial condition)}

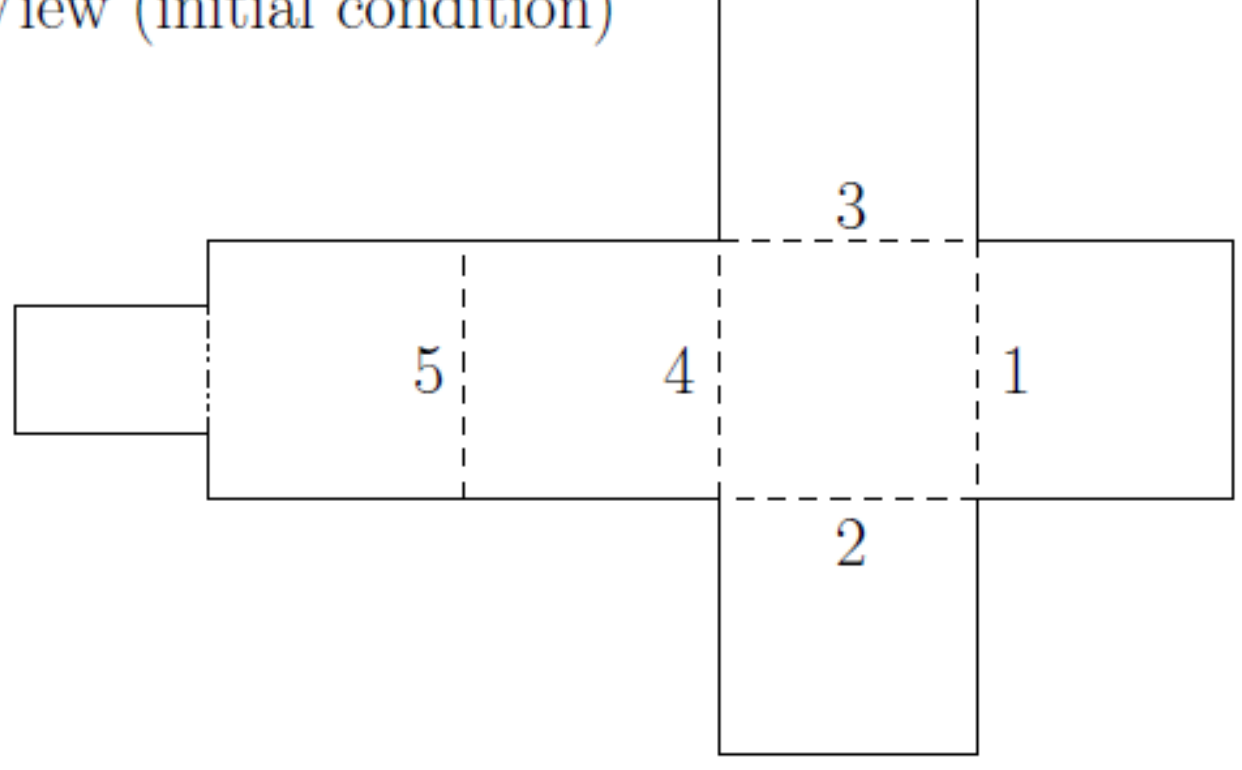

(a)

\section{Top View (during step 4)}
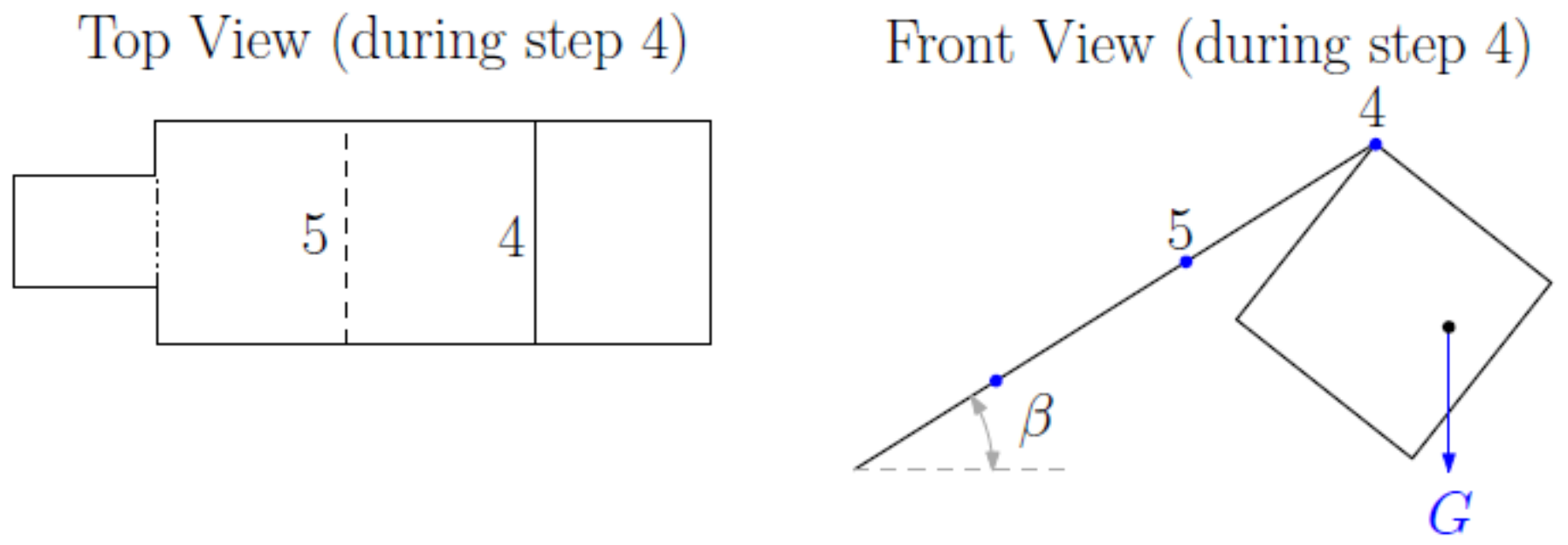

(b)

\section{Figure 11}

This figure plots the schematic for folding a closed box. (a) A at sheet is cut into the shape plotted by the solid lines; the dashed lines represent the folding path; the numbers with the creases indicate the folding order. (b) The robotic arm rotates the workpiece by an angle $\beta$ to form a closed box. 


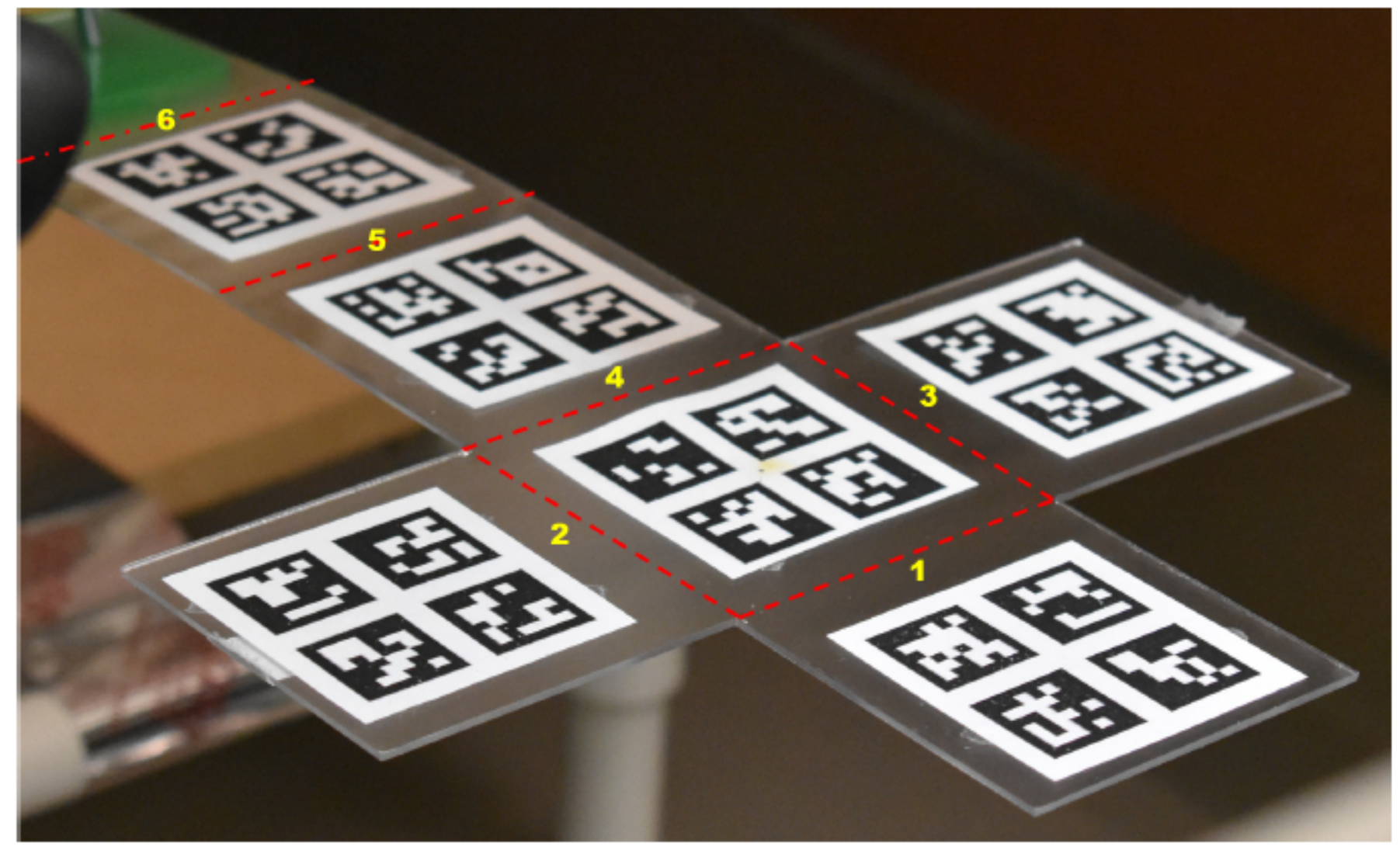

\section{Figure 12}

The laser-cut sheet precursor is clamped on the robotic arm. The dashed line segments $1 \sim 5$ represent the folding paths (creases to be formed) to form the box. The dash-dot line segment 6 represents the cutting path after the box is formed. 


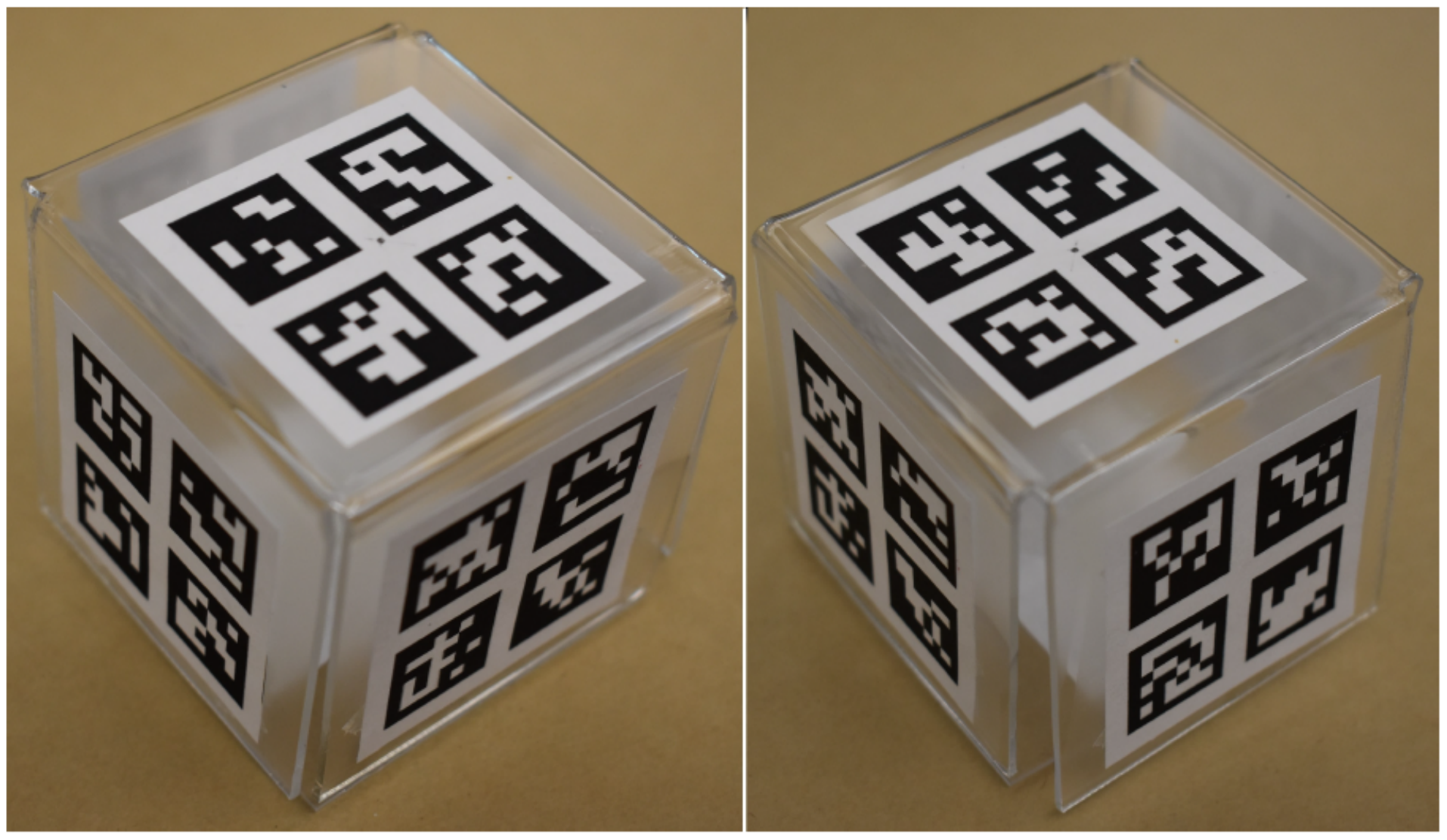

\section{Figure 13}

This figure presents a different viewpoint of the closed box that is manufactured by our laser kirigami platform. 